PROCEEDINGS OF THE

AMERICAN MATHEMATICAL SOCIETY

Volume 139, Number 4, April 2011, Pages 1155-116

S 0002-9939(2010)10765-7

Article electronically published on November 17, 2010

\title{
AN APPLICATION OF AMPLE VECTOR BUNDLES IN REAL ALGEBRAIC GEOMETRY
}

\author{
WOJCIECH KUCHARZ AND KAMIL RUSEK
}

(Communicated by Lev Borisov)

\begin{abstract}
Let $E$ be an algebraic vector bundle on a compact nonsingular real algebraic set $X$, and let $Z$ be the zero locus of a "generic" algebraic section of $E$. We investigate how certain cohomological invariants of $X$ and $Z$ are related. A crucial role in the proof is played by ample vector bundles on a suitable complexification of $X$.
\end{abstract}

\section{INTRODUCTION}

Let $X$ be a compact nonsingular real algebraic set (in $\mathbb{R}^{n}$ or $\mathbb{P}^{n}(\mathbb{R})$ for some $n$ ). A cohomology class in $H^{k}(X ; \mathbb{Z} / 2)$ is said to be algebraic if the homology class in $H_{l}(X ; \mathbb{Z} / 2)$ Poincaré dual to it can be represented by an $l$-dimensional algebraic subset of $X, l=\operatorname{dim} X-k$ (cf. [10] and [6, 9]). The set of all algebraic cohomology classes in $H^{k}(X ; \mathbb{Z} / 2)$ forms a subgroup denoted by $H_{\text {alg }}^{k}(X ; \mathbb{Z} / 2)$. The groups $H_{\mathrm{alg}}^{k}(-; \mathbb{Z} / 2)$ play a fundamental role in real algebraic geometry and have been extensively studied (cf. [2, 3, 4, 5, 6, 7, 15, 16, 18, 20, 24, and 9] for a short survey).

One can also associate with $X$ and any commutative ring $R$ other cohomological invariants of algebraic-geometric significance. A nonsingular projective complexification of $X$ is a pair $(V, e)$, where $V$ is a closed nonsingular subscheme of $\mathbb{P}_{\mathbb{R}}^{N}$ (for some $N$ ) and $e: X \rightarrow V(\mathbb{C})$ is an injective map such that $e(X)=V(\mathbb{R}), V(\mathbb{R})$ is Zariski dense in $V$, and $e$ induces a biregular isomorphism between $X$ and $V(\mathbb{R})$. Here the set $V(\mathbb{R})$ of real points of $V$ is regarded as a subset of the set $V(\mathbb{C})$ of complex points of $V$. Moreover, $V(\mathbb{R})$ is also viewed as an algebraic subset of $\mathbb{P}^{N}(\mathbb{R})$. The existence of $(V, e)$ follows from Hironaka's resolution of singularities [13. As is well known, $(V, e)$ is uniquely determined up to isomorphism over $\mathbb{R}$, provided that $\operatorname{dim} X=1$. However, if $\operatorname{dim} X \geq 2$, then $X$ admits infinitely many pairwise nonisomorphic projective complexifications, for $V$ can be blown up along a nonsingular center disjoint from $V(\mathbb{R})$. In view of this nonuniqueness, it is remarkable that the subgroup

$$
H_{\mathbb{C}}^{k}(X ; R):=e^{\star}\left(H^{k}(V(\mathbb{C}) ; R)\right)
$$

of $H^{k}(X ; R)$, where $e^{\star}: H^{k}(V(\mathbb{C}) ; R) \rightarrow H^{k}(X ; R)$ denotes the homomorphism induced by $e$, does not depend on the choice of $(V, e)$. This is proved in [22] for

Received by the editors February 5, 2010.

2010 Mathematics Subject Classification. Primary 14F05, 14F25, 14P05, 14P25.

Key words and phrases. Real algebraic set, algebraic vector bundle, algebraic cohomology class, nonsingular projective complexification, ample vector bundle.

(C)2010 American Mathematical Society 1155

Reverts to public domain 28 years from publication 
$X$ orientable over $R$, and in [11 for arbitrary $X$. Note that in both [22] and [11] the authors use different notation for our $H_{\mathbb{C}}^{k}(-; R)$. Properties and applications of $H_{\mathbb{C}}^{k}(-; R)$ are elaborated upon in [11, 17, 22, 23.

The groups $H_{\mathrm{alg}}^{k}(-; \mathbb{Z} / 2)$ and $H_{\mathbb{C}}^{k}(-; R)$ are subtle invariants that are, in general, hard to compute. In this paper we make use of ample vector bundles to estimate the size of $H_{\mathrm{alg}}^{k}(Z ; \mathbb{Z} / 2)$ and compute $H_{\mathbb{C}}^{k}(Z ; R)$ in terms of $H^{k}(X, \mathbb{Z} / 2)$ and $H_{\mathbb{C}}^{k}(X ; R)$, respectively, for a large class of algebraic subsets $Z$ of $X$. The ample vector bundles do not appear in the formulation of our result but only in the proof.

An algebraic vector bundle on $X$ is, by definition, isomorphic to an algebraic subbundle of the trivial vector bundle with total space $X \times \mathbb{R}^{q}$ for some $q$; cf. 6, Chapter 12] (such an object was called a strongly algebraic vector bundle in the earlier literature [4, 5, 7]). Thus the category of algebraic vector bundles on $X$ is isomorphic to the category of finitely generated projective modules over the ring of regular functions on $X$.

The zero locus of a section $s: X \rightarrow E$ of a vector bundle $E$ on $X$ will be denoted by $Z(s)$,

$$
Z(s)=\{x \in X \mid s(x)=0\} .
$$

Theorem 1.1. Let $X$ be a compact irreducible nonsingular real algebraic set and let $E$ be an algebraic vector bundle on $X$ with $d=\operatorname{dim} X-\operatorname{rank} E$ positive. For each algebraic section $s$ of $E$, there exist algebraic sections $s_{1}, \ldots, s_{p}$ of $E$ and a proper algebraic subset $\Sigma$ of $\mathbb{R}^{p}$ such that $s_{1}, \ldots, s_{p}$ generate $E$ and for each point $t=\left(t_{1}, \ldots, t_{p}\right)$ in $\mathbb{R}^{p} \backslash \Sigma$, the algebraic section $\sigma_{t}=s+t_{1} s_{1}+\cdots+t_{p} s_{p}$ is transverse to the zero section of $E$, and its zero locus $Z_{t}=Z\left(\sigma_{t}\right)$ is either empty or else it is a d-dimensional irreducible nonsingular algebraic subset of $X$ satisfying

$$
\begin{aligned}
& H_{\mathrm{alg}}^{k}\left(Z_{t} ; \mathbb{Z} / 2\right) \subseteq i_{t}^{\star}\left(H^{k}(X ; \mathbb{Z} / 2)\right) \quad \text { for } k<d / 2 \text {, } \\
& H_{\mathbb{C}}^{k}\left(Z_{t} ; R\right)=i_{t}^{\star}\left(H_{\mathbb{C}}^{k}(X ; R)\right) \quad \text { for } k<d,
\end{aligned}
$$

where $i_{t}: Z_{t} \hookrightarrow X$ is the inclusion map.

We postpone the proof until Section 2 and now derive the following approximation result for smooth (of class $\mathcal{C}^{\infty}$ ) submanifolds of $X$.

Corollary 1.2. Let $X$ be a compact irreducible nonsingular real algebraic set and let $E$ be an algebraic vector bundle on $X$ with $d=\operatorname{dim} X-\operatorname{rank} E$ positive. Let $M$ be a smooth submanifold of $X$ that is the zero locus of a smooth section of $E$ transverse to the zero section. Then there exists a smooth diffeomorphism $\varphi: X \rightarrow X$, arbitrarily close in the $\mathcal{C}^{\infty}$ topology to the identity map, such that $Z:=\varphi(M)$ is an irreducible nonsingular algebraic subset of $X$ satisfying

$$
\begin{aligned}
H_{\text {alg }}^{k}(Z ; \mathbb{Z} / 2) & \subseteq i^{\star}\left(H^{k}(X ; \mathbb{Z} / 2)\right) & & \text { for } k<d / 2, \\
H_{\mathbb{C}}^{k}(Z ; R) & =i^{\star}\left(H_{\mathbb{C}}^{k}(X ; R)\right) & & \text { for } k<d,
\end{aligned}
$$

where $i: Z \hookrightarrow X$ is the inclusion map.

Proof. Let $w: X \rightarrow E$ be a smooth section transverse to the zero section and satisfying $Z(w)=M$. There exists an algebraic section $s: X \rightarrow E$ arbitrarily close in the $\mathcal{C}^{\infty}$ topology to $w$ (cf. [6, Theorem 12.3.2]). For this section $s$, let $s_{1}, \ldots, s_{p}$ and $\Sigma$ be as in Theorem 1.1. If $t$ in $\mathbb{R}^{p} \backslash \Sigma$ is close to $0 \in \mathbb{R}^{p}$, then the algebraic section $\sigma_{t}=s+t_{1} s_{1}+\cdots+t_{p} s_{p}$ of $E$ is close to $w$ in the $\mathcal{C}^{\infty}$ topology. According to [1, Theorem 20.2], there exists a smooth diffeomorphism $\varphi: X \rightarrow X$, close in the $\mathcal{C}^{\infty}$ topology to the identity map, with $\varphi(M)=Z_{t}$. It suffices to set $Z=Z_{t}$. 
In Theorem 1.1 and Corollary 1.2 the cohomology groups of $X$ tell us nothing about the cohomology groups of $Z$ and $Z_{t}$. We obtain interesting relationships only after passing to $H_{\text {alg }}^{k}(-; \mathbb{Z} / 2)$ and $H_{\mathbb{C}}^{k}(-; R)$. These last two groups have the expected functorial property: If $f: X \rightarrow Y$ is a regular map between compact nonsingular real algebraic sets, then

$$
f^{\star}\left(H_{\mathrm{alg}}^{k}(Y ; \mathbb{Z} / 2)\right) \subseteq H_{\mathrm{alg}}^{k}(X ; \mathbb{Z} / 2) \quad \text { and } \quad f^{\star}\left(H_{\mathbb{C}}^{k}(Y ; R)\right) \subseteq H_{\mathbb{C}}^{k}(X ; R)
$$

(cf. [10, Section 5] or [2, 5] for the former inclusion and [22, 11] for the latter). In particular, with notation as in Theorem 1.1, the inclusions

$$
\begin{aligned}
i_{t}^{\star}\left(H_{\mathrm{alg}}^{k}(X ; \mathbb{Z} / 2)\right) & \subseteq H_{\mathrm{alg}}^{k}\left(Z_{t} ; \mathbb{Z} / 2\right), \\
H_{\mathbb{C}}^{k}(Z ; R) & \supseteq i^{\star}\left(H_{\mathbb{C}}^{k}(X ; R)\right)
\end{aligned}
$$

are automatically satisfied for all $k$. An analogous remark remains valid in the context of Corollary 1.2 .

Conjecture 1.3. In Theorem 1.1 and Corollary 1.2 ,

$$
H_{\text {alg }}^{k}\left(Z_{t} ; \mathbb{Z} / 2\right)=i_{t}^{\star}\left(H_{\text {alg }}^{k}(X ; \mathbb{Z} / 2)\right) \text { for } k<d / 2
$$

and

$$
H_{\mathrm{alg}}^{k}(Z ; \mathbb{Z} / 2)=i^{\star}\left(H_{\text {alg }}^{k}(X ; \mathbb{Z} / 2)\right) \quad \text { for } k<d / 2,
$$

respectively.

In view of the functoriality of $H_{\mathrm{alg}}^{k}(-; \mathbb{Z} / 2)$, Conjecture 1.3 is true if

$$
H_{\text {alg }}^{k}(X ; \mathbb{Z} / 2)=H^{k}(X ; \mathbb{Z} / 2) \text { for } k<d / 2 .
$$

Theorem 1.1 and Corollary 1.2 are applicable, and Conjecture 1.3 is true in the following two cases.

Example 1.4. Each topological real vector bundle on $\mathbb{P}^{n}(\mathbb{R})$ is isomorphic to an algebraic vector bundle, and

$$
H_{\text {alg }}^{k}\left(\mathbb{P}^{n}(\mathbb{R}) ; \mathbb{Z} / 2\right)=H^{k}\left(\mathbb{P}^{n}(\mathbb{R}) ; \mathbb{Z} / 2\right) \quad \text { for all } k
$$

(cf. [6, Example 12.3.7c, Proposition 11.3.3]).

Example 1.5. Let $\mathbb{M}_{n}(\mathbb{R}) \cong \mathbb{R}^{n^{2}}$ denote the space of real $n \times n$ matrices. Given a matrix $A$, we write ${ }^{\mathrm{t}} A$ for its transpose. The real Grassmannian $\mathbb{G}_{n, r}(\mathbb{R})$ of $r$-dimensional vector subspaces of $\mathbb{R}^{n}$ can be identified with the algebraic subset

$$
\left\{A \in \mathbb{M}_{n}(\mathbb{R}) \mid A={ }^{\mathrm{t}} A, A^{2}=A, \operatorname{trace} A=r\right\}
$$

of $\mathbb{M}_{n}(\mathbb{R})$. The universal vector bundle $E_{n, r}$ on $\mathbb{G}_{n, r}(\mathbb{R})$ is algebraic. Moreover, $\mathbb{G}_{n, r}(\mathbb{R})$ is nonsingular and

$$
H_{\text {alg }}^{k}\left(\mathbb{G}_{n, r}(\mathbb{R}) ; \mathbb{Z} / 2\right)=H^{k}\left(\mathbb{G}_{n, r}(\mathbb{R}) ; \mathbb{Z} / 2\right) \quad \text { for all } k .
$$

For these facts one can consult [6, pp. 71, 272, 303].

Corollary 1.2 has an interesting application even when $E$ is a trivial vector bundle. First recall that every compact smooth manifold $M$ is diffeomorphic to a nonsingular real algebraic set, called an algebraic model of $M$; cf. [26] or [6, Theorem 14.1.10] (and also [21] for a weaker but influential result). How the groups $H_{\text {alg }}^{k}(Y ; \mathbb{Z} / 2)$ vary as $Y$ runs through the class of all algebraic models of $M$ is a challenging problem (cf. [5, 7, 16, 17]). 
Corollary 1.6. Let $M$ be an $m$-dimensional smooth manifold that is the boundary of a compact smooth parallelizable manifold with boundary. If $m \geq 1$, then $M$ has an irreducible algebraic model $Y$ satisfying

$$
H_{\text {alg }}^{k}(Y ; \mathbb{Z} / 2)=0 \quad \text { for } 1 \leq k<m / 2 .
$$

Proof. Let $M=\partial Q$, where $Q$ is a compact smooth parallelizable manifold. We may assume that $Q$ is a smooth submanifold of the unit sphere $\mathbb{S}^{n}, n \geq 2 \operatorname{dim} Q+1$. Since the tangent bundle of $Q$ is trivial, the normal bundle of $Q$ in $\mathbb{S}^{n}$ is stably trivial, and hence trivial being of rank greater than $\operatorname{dim} Q$ (cf. [14, p. 100, Theorem 1.5]). According to [8, Theorem 1.12] there exists a smooth map $f: \mathbb{S}^{n} \rightarrow \mathbb{R}^{n-m}$ for which $0 \in \mathbb{R}^{n-m}$ is a regular value and $M=f^{-1}(0)$. Applying Corollary 1.2 to the trivial vector bundle on $\mathbb{S}^{n}$ with total space $\mathbb{S}^{n} \times \mathbb{R}^{n-m}$, we obtain an irreducible nonsingular algebraic subset $Y$ of $\mathbb{S}^{n}$ that is diffeomorphic to $M$ and satisfies

$$
H_{\text {alg }}^{k}(Y ; \mathbb{Z} / 2) \subseteq i^{\star}\left(H^{k}\left(\mathbb{S}^{n} ; \mathbb{Z} / 2\right)\right) \quad \text { for } k<m / 2 .
$$

The proof is complete.

Let $N$ be a compact smooth stably parallelizable manifold. Then the smooth manifold $M=(N \times\{0\}) \cup(N \times\{1\})$, which is the disjoint union of two copies of $N$, satisfies the hypothesis of Corollary [1.6] The same is true for $M=N \times \mathbb{S}^{1}$.

\section{VECTOR BUNDLES ON SCHEMES OVER $\mathbb{R}$}

Let $V$ be a closed nonsingular subscheme of $\mathbb{P}_{\mathbb{R}}^{n}$ (for some $n$ ) with $V(\mathbb{R})$ nonempty. We regard $V(\mathbb{R})$ and $V(\mathbb{C})$ as algebraic subsets of $\mathbb{P}^{n}(\mathbb{R})$ and $\mathbb{P}^{n}(\mathbb{C})$, respectively. Each vector bundle $\mathcal{E}$ on $V$ gives rise to algebraic vector bundles $\mathcal{E}(\mathbb{R})$ on $V(\mathbb{R})$ and $\mathcal{E}(\mathbb{C})$ on $V(\mathbb{C})$. For any section $v: V \rightarrow \mathcal{E}$, the corresponding sections

$$
v(\mathbb{R}): V(\mathbb{R}) \rightarrow \mathcal{E}(\mathbb{R}) \quad \text { and } \quad v(\mathbb{C}): V(\mathbb{C}) \rightarrow \mathcal{E}(\mathbb{C})
$$

are algebraic. We say that the section $v$ is transverse regular if the section $v(\mathbb{C})$ is transverse to the zero section of $\mathcal{E}(\mathbb{C})$. The zero scheme of $v$ will be denoted by $Z(v)$. We consider $V_{\mathbb{C}}=V \times_{\mathbb{R}} \mathbb{C}$ as a scheme over $\mathbb{C}$ and denote by $\mathcal{E}_{\mathbb{C}}$ the vector bundle on $V_{\mathbb{C}}$ determined by $\mathcal{E}$. Similarly, we denote by $v_{\mathbb{C}}: V_{\mathbb{C}} \rightarrow \mathcal{E}_{\mathbb{C}}$ the section determined by $v$. If $\mathcal{E}$ is generated by the sections $v_{1}, \ldots, v_{p}$, then $\mathcal{E}_{\mathbb{C}}$ is generated by the sections $v_{1} \mathbb{C}, \ldots, v_{p} \mathbb{C}$.

Proof of Theorem 1.1. We may assume that $X=V(\mathbb{R})$ and $E=\mathcal{E}(\mathbb{R})$, where $V$ is a closed irreducible nonsingular subscheme of $\mathbb{P}^{n}(\mathbb{R})$ and $\mathcal{E}$ is a vector bundle on $V$ (cf. [15, Lemma 3.4]). There exist an open neighborhood $V_{0}$ of $X$ in $V$ and a section $v_{0}: V_{0} \rightarrow E$ which is an extension of $s$, that is, $v_{0}(\mathbb{R}): V_{0}(\mathbb{R})=X \rightarrow \mathcal{E}(\mathbb{R})=E$ is equal to $s$. We have

$$
V_{0}=V \backslash Z\left(H_{1}, \ldots, H_{l}\right),
$$

where the $H_{j}$ are homogeneous polynomials in $\mathbb{R}\left[T_{0}, \ldots, T_{n}\right]$ and $Z\left(H_{1}, \ldots, H_{l}\right)$ is the closed subset of $\mathbb{P}_{\mathbb{R}}^{n}$ determined by the equations $H_{1}=0, \ldots, H_{l}=0$. Set $r_{j}=\operatorname{deg} H_{j}, r=\max \left\{r_{1}, \ldots, r_{l}\right\}$, and

$$
H=\sum_{j=1}^{l}\left(T_{0}^{2}+\cdots+T_{n}^{2}\right)^{r-r_{j}} H_{j}^{2} .
$$

Then $H$ is a homogeneous polynomial of degree $2 r$ and

$$
V(\mathbb{R}) \subseteq V \backslash Z(H) \subseteq V_{0}
$$


where $Z(H)$ is the closed subset of $\mathbb{P}_{\mathbb{R}}^{n}$ determined by the equation $H=0$.

For each integer $c$, we have the vector bundle $\mathcal{O}(c)$ on $\mathbb{P}_{\mathbb{R}}^{n}$. Let $h: \mathbb{P}_{\mathbb{R}}^{n} \rightarrow \mathcal{O}(2 r)$ be the section of $\mathcal{O}(2 r)$ determined by the homogeneous polynomial $H$. Note that

$$
Z(h)=Z(H) .
$$

Let $\mathcal{L}=\left.\mathcal{O}(2 r)\right|_{V}$ and $u=\left.h\right|_{V}$. Then $\mathcal{L}$ is an ample line bundle on $V$ and $u: V \rightarrow \mathcal{L}$ is a section. By construction,

$$
V(\mathbb{R}) \subseteq V \backslash Z(u)=V \backslash Z(H) \subseteq V_{0} .
$$

Given a positive integer $m$, we set $\mathcal{E}(m)=\mathcal{E} \otimes \mathcal{L}^{m}$, where $\mathcal{L}^{m}=\mathcal{L} \otimes \cdots \otimes \mathcal{L}$ is the $m$-fold tensor product. There exists a positive integer $m_{0}$ such that for each integer $m \geq m_{0}$, the vector bundle $\mathcal{E}(m)$ is generated by global sections (cf. [12, p. 153]) and the section

$$
v_{0} \otimes u^{m}: V \backslash Z(u) \rightarrow \mathcal{E}(m),
$$

where $u^{m}=u \otimes \cdots \otimes u: V \rightarrow \mathcal{L}^{m}$, can be extended to a section $w_{m}: V \rightarrow \mathcal{E}(m)$ (cf. [12, Lemma 5.14]). We may also assume that $\mathcal{E}(m)$ is an ample vector bundle.

Fix $m \geq m_{0}$ and set $v=w_{m}$. Let $v_{1}, \ldots, v_{p}$ be sections of $\mathcal{E}(m)$ that generate $\mathcal{E}(m)$. Given $t=\left(t_{1}, \ldots, t_{p}\right)$ in $\mathbb{R}^{p}$, set

$$
\tau_{t}=v+t_{1} v_{1}+\cdots+t_{p} v_{p} \quad \text { and } \quad V_{t}=Z\left(\tau_{t}\right) .
$$

Since the sections $v_{1 \mathbb{C}}, \ldots, v_{p} \mathbb{C}$ generate $\mathcal{E}(m)_{\mathbb{C}}$, there exists a proper algebraic subset $\Sigma$ of $\mathbb{R}^{p}$ such that for each point $t$ in $\mathbb{R}^{p} \backslash \Sigma$, the section $\tau_{t}$ is transverse regular.

Henceforth we fix $t$ in $\mathbb{R}^{p} \backslash \Sigma$. Then the subscheme $V_{t}$ of $V$ is nonsingular of dimension $d$. According to Sommese's theorem (cf. [25, Proposition 1.16] or [19, Theorem 7.1.1]),

$$
H^{k}\left(V(\mathbb{C}), V_{t}(\mathbb{C}) ; \mathbb{Z}\right)=0 \quad \text { for } k \leq d .
$$

Taking $k=1$, we obtain that $V_{t}(\mathbb{C})$ is connected, and hence $V_{t}$ is irreducible. Moreover, if $j_{t}: V_{t} \hookrightarrow V$ is the inclusion morphism, then

$$
j_{t}(\mathbb{C})^{\star}: H^{k}(V(\mathbb{C}) ; R) \rightarrow H^{k}\left(V_{t}(\mathbb{C}) ; R\right) \quad \text { is an isomorphism for } k<d .
$$

Suppose that the algebraic subset $V_{t}(\mathbb{R})$ of $V(\mathbb{R})$ is nonempty. Then

$$
V_{t}(\mathbb{R}) \text { is irreducible and nonsingular, } \operatorname{dim} V_{t}(\mathbb{R})=d \text {. }
$$

We will now deal with $H_{\text {alg }}^{k}(-; \mathbb{Z} / 2)$. According to [18, Theorem 2.1], condition (11) (with $R=\mathbb{Z}$ ) implies that

$$
H_{\text {alg }}^{k}\left(V_{t}(\mathbb{R}) ; \mathbb{Z} / 2\right) \subseteq j_{t}(\mathbb{R})^{\star}\left(H^{k}(V(\mathbb{R}) ; \mathbb{Z} / 2)\right) \quad \text { for } k<d / 2 .
$$

In order to analyze $H_{\mathbb{C}}^{k}(-; R)$, let $e: V(\mathbb{R}) \hookrightarrow V(\mathbb{C})$ and $e_{t}: V_{t}(\mathbb{R}) \hookrightarrow V_{t}(\mathbb{C})$ be the inclusion maps. The following diagram is commutative:

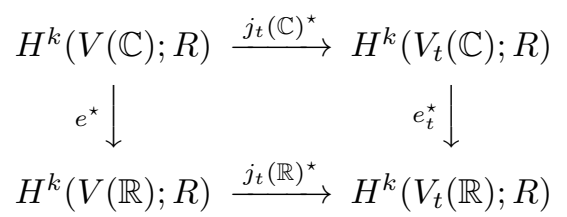

Since $(V, e)$ and $\left(V_{t}, e_{t}\right)$ are nonsingular projective complexifications of $V(\mathbb{R})$ and $V_{t}(\mathbb{R})$, respectively, condition (11) implies that

$$
H_{\mathbb{C}}^{k}\left(V_{t}(\mathbb{R}) ; R\right)=j_{t}(\mathbb{R})^{\star}\left(H_{\mathbb{C}}^{k}(V(\mathbb{R}) ; R)\right) \quad \text { for } k<d .
$$


We are ready for the final step in the proof. Since the zero locus of the section of $\mathcal{O}(2 r)$ determined by the polynomial $\left(T_{0}^{2}+\cdots+T_{n}^{2}\right)^{r}$ does not intersect $\mathbb{P}^{n}(\mathbb{R})$, the algebraic vector bundle $\mathcal{O}(2 r)(\mathbb{R})$ on $\mathbb{P}^{n}(\mathbb{R})$ is algebraically trivial, and hence so is the algebraic vector bundle $\mathcal{L}(\mathbb{R})$ on $V(\mathbb{R})$. Consequently, the algebraic vector bundles $\mathcal{E}(m)(\mathbb{R})$ and $\mathcal{E}(\mathbb{R})=E$ on $V(\mathbb{R})=X$ are algebraically isomorphic. If $\Phi: \mathcal{E}(m)(\mathbb{R}) \rightarrow E$ is an algebraic isomorphism, then $\Phi \circ v(\mathbb{R})=f s$ for some regular function $f: X \rightarrow \mathbb{R}$ with $f^{-1}(0)=\emptyset$. Let $\pi: \mathcal{E}(m)(\mathbb{R}) \rightarrow X$ be the bundle projection and $\Psi=(1 / f \circ \pi) \Phi$. Then $\Psi: \mathcal{E}(m)(\mathbb{R}) \rightarrow E$ is an algebraic isomorphism satisfying $\Psi \circ v(\mathbb{R})=s$. Since the sections $v_{1}, \ldots, v_{p}$ generate $\mathcal{E}(m)$, the algebraic sections $s_{1}:=\Psi \circ v_{1}(\mathbb{R}), \ldots, s_{p}:=\Psi \circ v_{p}(\mathbb{R})$ generate $E$. We have $\Psi \circ \tau_{t}(\mathbb{R})=\sigma_{t}$, where $\sigma_{t}=s+t_{1} s_{1}+\cdots+t_{p} s_{p}$. The transverse regularity of $\tau_{t}$ implies that $\sigma_{t}$ is

transverse to the zero section of $E$. By construction, $Z\left(\sigma_{t}\right)=Z\left(\tau_{t}(\mathbb{R})\right)=V_{t}(\mathbb{R})$. The proof is complete in view of (2), (3), and (4).

\section{REFERENCES}

[1] R. Abraham and J. Robbin, Transversal Mappings and Flows, New York, Benjamin, 1967. MR0240836 (39:2181)

[2] S. Akbulut and H. King, Submanifolds and homology of nonsingular algebraic varieties, Amer. J. Math., 107 (1985), 45-83. MR778089 (86m:14017)

[3] S. Akbulut and H. King, Topology of Real Algebraic Sets, Math. Sci. Research Institute Publ. 25, Springer, 1992. MR 1225577 (94m:57001)

[4] R. Benedetti and A. Tognoli, Approximation theorems in real algebraic geometry, Boll. Unione Mat. Ital., Suppl., 2 (1980), 209-228. MR675502 (84h:58004)

[5] R. Benedetti and A. Tognoli, Remarks and counterexamples in the theory of real vector bundles and cycles. In: Géométrie algébrique réelle et formes quadratiques, Lecture Notes in Math. 959, Springer, 1982, 198-211. MR683134(85a:14018)

[6] J. Bochnak, M. Coste and M.-F. Roy, Real Algebraic Geometry, Ergeb. der Math und ihrer Grenzgeb. Folge 3, Vol. 36, Berlin-Heidelberg-New York, Springer, 1998. MR 1659509 (2000a:14067)

[7] J. Bochnak and W. Kucharz, Algebraic models of smooth manifolds, Invent. Math., 97 (1989), 585-611. MR.1005007 (91b:14076)

[8] J. Bochnak and W. Kucharz, Complete intersections in differential topology and algebraic geometry, Boll. Unione Mat. Ital. (7), 10-B (1996), 1019-1041. MR.1430164 (98b:57045)

[9] J. Bochnak and W. Kucharz, On homology classes represented by real algebraic varieties, Banach Center Publications, Vol. 44, 21-35, Warsaw, 1998. MR.1677394 (2000b:14080)

[10] A. Borel and A. Haefliger, La classe d'homologie fondamentale d'un espace analytique, Bull. Soc. Math. France 89 (1961), 461-513. MR0149503 (26:6990)

[11] S. Dolega, Complexification and cohomology in real algebraic geometry, Ph.D. Thesis, University of New Mexico, 2004.

[12] R. Hartshorne, Algebraic Geometry, Graduate Texts in Math., Vol. 33, New York-HeidelbergBerlin, Springer, 1977. MR0463157 (57:3116)

[13] H. Hironaka, Resolution of singularities of an algebraic variety over a field of characteristic zero, Ann. of Math., 79 (1964), 109-326. MR0199184 (33:7333)

[14] D. Husemoller, Fibre Bundles, New York, Springer, 1994. MR,1249482 (94k:55001)

[15] W. Kucharz, Homology classes of real algebraic sets, Ann. Inst. Fourier (Grenoble), 58 (2008), 989-1022. MR2427517 (2009f:14118)

[16] W. Kucharz, Cycles on algebraic models of smooth manifolds, J. Eur. Math. Soc., 11 (2009), 393-405. MR2486938 (2010h:14094)

[17] W. Kucharz and K. Kurdyka, Complexification of algebraic models of smooth manifolds, to appear in J. London Math. Soc.

[18] W. Kucharz and J. van Hamel, Transcendental manifolds in projective space and StiefelWhitney classes, Ann. Sc. Norm. Super. Pisa Cl. Sci. (5) 8 (2009), 267-277. MR2548247 (2010g:14082)

[19] R. Lazarsfeld, Positivity in Algebraic Geometry. I, Ergeb. der Math. und ihrer Grenzgeb. Folge 3, Vol. 48, Berlin-Heidelberg, Springer, 2004. MR2095471 (2005k:14001a) 
[20] F. Mangolte, Cycles algébriques et topologie des surfaces bielliptiques réelles, Comment. Math. Helv., 78 (2003), 385-393. MR1988202 (2005b:14020)

[21] J. Nash, Real algebraic manifolds, Ann. of Math. (2), 56 (1952), 405-421. MR0050928 $(14: 403 \mathrm{~b})$

[22] Y. Ozan, On homology of real algebraic varieties, Proc. Amer. Math. Soc., 129 (2001), 31673175. MR 1844989 (2002m:14048)

[23] Y. Ozan, Relative topology of real algebraic varieties in their complexifications, Pacific J. Math, 217 (2004), 291-302. MR2109937 (2005i:57036)

[24] R. Silhol, Real Algebraic Surfaces, Lecture Notes in Math. 1392, Springer, 1989. MR.1015720 (91i:14045)

[25] A. Sommese, Submanifolds of Abelian varieties, Math. Ann., 233 (1978), 229-256. MR0466647 (57:6524)

[26] A. Tognoli, Su una cognettura di Nash, Ann. Scuola Norm. Sup. Pisa, Sci. Fis. Mat. (3), 27 (1973), 167-185. MR0396571 (53:434)

Institute of Mathematics, Jagiellonian University, ul. Profesora Łojasiewicza 6, 30-348 KRAKÓw, POLAND

E-mail address: Wojciech.Kucharz@im.uj.edu.pl

Institute of Mathematics, Jagiellonian University, Ul. Profesora Łojasiewicza 6, 30-348 Kraków, POLAND

E-mail address: Kamil.Rusek@im.uj.edu.pl 